\title{
Review Article \\ MMSET: Role and Therapeutic Opportunities in Multiple Myeloma
}

\author{
Zhigang Xie ${ }^{1}$ and Wee Joo Chng ${ }^{1,2,3}$ \\ ${ }^{1}$ Cancer Science Institute of Singapore, National University of Singapore, 14 Medical Drive, Singapore 117599 \\ ${ }^{2}$ Department of Medicine, Yong Loo Lin School of Medicine, National University of Singapore, 1E Kent Ridge Road, Singapore 119228 \\ ${ }^{3}$ Department of Haematology-Oncology, National University Cancer Institute of Singapore, National University Health System, \\ Singapore 119228
}

Correspondence should be addressed to Wee Joo Chng; mdccwj@nus.edu.sg

Received 17 March 2014; Accepted 16 June 2014; Published 1 July 2014

Academic Editor: Dong Soon Lee

Copyright (C) 2014 Z. Xie and W. J. Chng. This is an open access article distributed under the Creative Commons Attribution License, which permits unrestricted use, distribution, and reproduction in any medium, provided the original work is properly cited.

\begin{abstract}
Recurrent chromosomal translocations are central to the pathogenesis, diagnosis, and prognosis of hematologic malignancies. The translocation $\mathrm{t}(4 ; 14)(\mathrm{p} 16 ; \mathrm{q} 32)$ is one of the most common translocations in multiple myeloma $(\mathrm{MM})$ and is associated with very poor prognosis. The $t(4 ; 14)$ translocation leads to the simultaneous overexpression of two genes, FGFR3 (fibroblast growth factor receptor 3) and MMSET (multiple myeloma SET domain), both of which have potential oncogenic activity. However, approximately $30 \%$ of $\mathrm{t}(4 ; 14) \mathrm{MM}$ patients do not express FGFR3 and have poor prognosis irrespective of FGFR3 expression, whereas MMSET overexpression is universal in $\mathrm{t}(4 ; 14)$ cases. In this review, we provide an overview of recent findings regarding the oncogenic roles of MMSET in MM and its functions on histone methylation. We also highlight some of MMSET partners and its downstream signalling pathways and discuss the potential therapeutics targeting MMSET.
\end{abstract}

\section{Introduction}

Compelling data has emerged that epigenetic changes underlie a wide variety of pathologies, including cancer $[1,2]$. Epigenetic regulation includes DNA methylation and covalent histone modifications. These processes may play an important role in the initiation and progression of many cancers, including the haematological malignancy multiple myeloma (MM).

MMSET (multiple myeloma SET domain), also known as Wolf-Hirschhorn syndrome candidate 1 (WHSC1) or nuclear receptor-binding SET domain 2 (NSD2), is a member of the NSD histone methyltransferase (HMT) family also including NSD1 and NSD3 [3-5]. The MMSET gene spans $120 \mathrm{~kb}$, consists of 24 exons, and undergoes complex alternative splicing. Two major transcripts were identified: type I encodes a protein of 647 amino acids and type II encodes a protein of 1365 amino acids. Both proteins share a common amino terminus [6]. A third transcript initiated within a middle intron of MMSET encodes a protein named RE-IIBP
[7] (Figure 1). Conserved domain architecture analysis indicated that MMSET is a multidomain protein containing a set domain (determining protein lysine methyltransferase activity), 2 PWWP (named for a conserved Pro-Trp-TrpPro motif) domains, a HMG (high mobility group) box, and 3 PHD (plant homeodomain) fingers (Figure 1). The PWWP, HMG, and PHD domains are responsible for nuclear localization, DNA-binding, and recognition of histone marks [8-11]. The importance of MMSET in malignancy was first highlighted by characterization of the $t(4 ; 14)$ translocation in about $15 \%$ of MM, which fuses the MMSET gene to the immunoglobulin heavy-chain promoter/enhancer, leading to dramatic upregulation of MMSET expression $[6,12]$.

\section{Oncogenic Role of MMSET in MM}

Recurrent chromosomal translocations are central to the pathogenesis, diagnosis, and prognosis of hematologic malignancies. In the past decade, it has become apparent that approximately $50 \%$ of $\mathrm{MM}$ harbor recurrent translocations 


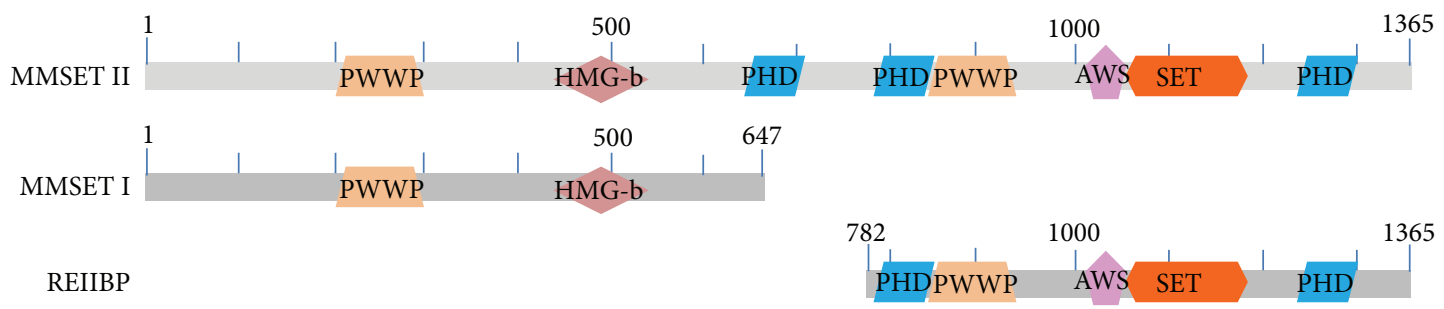

FIGURE 1: Schematic primary structure of three major MMSET isoforms. PWWP, named for a conserved Pro-Trp-Trp-Pro motif; HMGb, high mobility group (HMG) box domain; PHD, plant homeodomain zinc finger; AWS, associated with SET domain; and SET, lysine methyltransferase catalytic domain. Conserved domain architecture was analyzed through http://blast.ncbi.nlm.nih.gov.

involving the immunoglobulin heavy chain $(\operatorname{IgH})$ locus on chromosome $14 \mathrm{q} 32$ [13-15]. The translocation $\mathrm{t}(4 ; 14)(\mathrm{p} 16$; q32) is one of the most common translocations in MM, affecting $15 \%$ of patients, and is associated with very poor prognosis [16]. The $t(4 ; 14)$ translocation leads to the simultaneous overexpression of two genes, FGFR3 (fibroblast growth factor receptor 3) and MMSET. FGFR3 has transforming activity in vitro and in vivo, but approximately $30 \%$ of $\mathrm{t}(4 ; 14)$ MM patients do not express FGFR3, whereas overexpression of MMSET isoforms is a universal feature of $t(4 ; 14)$ cases $[12,17,18]$. Furthermore, the poor prognosis of $t(4 ; 14)$ persists irrespective of FGFR3 expression [12]. These data suggest that MMSET may be the critical oncogene in this translocation. Downregulation of MMSET expression in MM cell lines indicated that $\mathrm{t}(4 ; 14) \mathrm{MM}$ cells rely on MMSET expression for clonogenic growth and tumorigenicity in vivo [13]. These results provide the first direct evidence that MMSET is an oncogene and plays a significant role in $\mathrm{t}(4 ; 14) \mathrm{MM}$. Later several groups reported that depletion of MMSET could also inhibit proliferation and induce cell cycle arrest and apoptosis [19-22]. Consistent with MMSET knockdown results, wild type MMSET, but not the MMSET catalytic mutants, could restore proliferation and colony formation of $t(4 ; 14) \mathrm{MM}$ cells upon MMSET deletion. Furthermore, complementation of MMSET knockout cells with wild type MMSET instead of catalytic mutants could restore their tumorigenicity in vivo $[10,22]$. These data further conformed that MMSET is an oncogene and its oncogenic role is dependent on its catalytic activity. Recent study has shown that MMSET mRNA level is also upregulated in 15 of 40 tumor types compared to their normal tissue counterparts. Furthermore, MMSET mRNA levels are associated with tumor aggressiveness or prognosis in several of these tumors [23]. Thus, in addition to $t(4 ; 14)$ MM, MMSET may contribute to the development of other cancer types.

\section{MMSET Function on Histone Methylation}

Histones are the stage of diverse posttranslational modifications that ultimately regulates the gene transcription. Lysine methylation is one prominent feature of the posttranslational histone modifications in the regulation of chromatin structure and function. Lysine-HMTs target specific histone residues on $\mathrm{H} 3$ and $\mathrm{H} 4$ and can transfer one, two, or three methyl groups on specific lysines on the histone tails
[24]. The MMSET protein contains AWS (associated with set) SET-PostSET domains that are highly conserved with yeast H3K36-specific methyltransferase Set2 [25]. It is first reported that the MMSET protein is a H4K20 methyltransferase with characteristics of a transcriptional corepressor [19]. Later, several reports suggested that MMSET could generate numerous different histone marks, including H3K4me2, H3K9me2, H3K27me3, H3K36me2, H3K36me3, and H4K20me2 [26-30]. Biologically, MMSET is reported to repress transcription through generation of H4K20me3 [19], $\mathrm{H} 3 \mathrm{~K} 27 \mathrm{me} 3$ [27], or H3K36me3 [29], to enhance transcription through generation of H3K4me2 [26] and H3K36me2 [21], and to mediate accumulation of 53BP1 to DNA damage sites through generation of H4K20me2 [30]. Recently multiple independent biochemical and cellular approaches were used to investigate and resolve the discrepancies regarding MMSET enzymatic activity. It is suggested that the principal physiologic activity of MMSET at chromatin is dimethylation of $\mathrm{H} 3 \mathrm{~K} 36$, and in the process rules out generation of H3K36me3, H4K20me2, and several other putative methyl products of MMSET [22]. This conclusion is in agreement with a study reporting in vitro dimethylation activity at H3K36 by the three NSD family members NSD1, NSD2, and NSD3/WHSC1L1 [28].

\section{MMSET Interacting Proteins and Downstream Targets}

A preliminary step in understanding protein structure and function is to determine which proteins interact with each other, thereby identifying the relevant biological pathways. MMSET is a multidomain protein containing a catalytic set domain and other highly conserved domains, such as PWWP, $\mathrm{PHD}$, and HMG-box, to mediate chromatin interaction and recognition of histone marks. Coprecipitation experiments indicated that MMSET could interact with histone modifiers HDAC1, HDAC2, and LSD1 [19, 31]. SET domain containing methyltransferases seems to be particularly sensitive to the sequence and posttranslational modifications surrounding the target lysine site [32]. Hence, the histone modification signature of MMSET is complex and might be mediated both by direct action of MMSET and by interplay with other histone modifiers. To determine the genes regulated by MMSET, Martinez-Garcia et al. profiled gene expression 


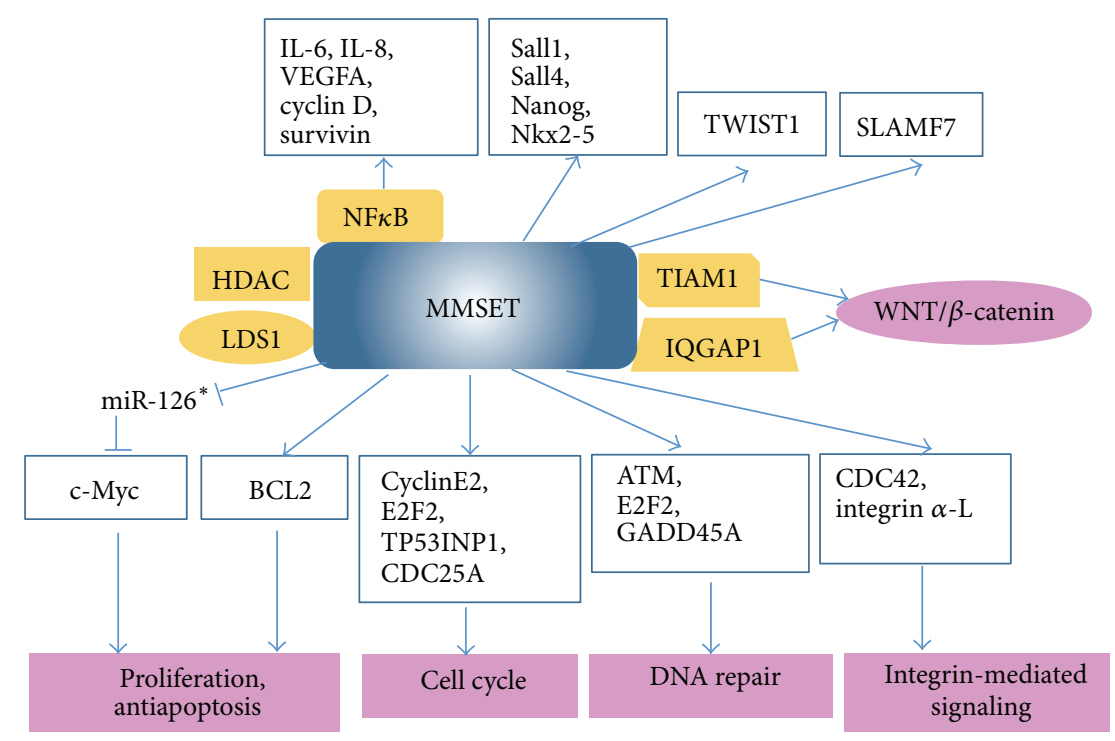

FIGURE 2: MMSET interacting proteins and downstream targets. MMSET interacts with its partners and activates oncogenic signalling pathways, which establish causal roles for MMSET in driving cancer initiation, development, and survival. Orange boxes: MMSET interacting proteins. White boxes: MMSET target genes.

in the loss-of-function (MMSET knockdown) and gain-offunction (MMSET reexpression in the MMSET knockout cell line) systems [21]. Signaling pathway analysis indicated that MMSET could regulate cell death and the p53 pathway (e.g., BAX, BCL2, and caspase 6), the cell cycle (cyclin E2, E2F2, TP53INP1, and CDC25A), genes for DNA repair (ATM, E2F2, and GADD45A), and integrin-mediated signaling (CDC42 and integrin alpha-L). These results are consistent with phenotypes of proliferation inhibition and apoptosis induction upon MMSET knockdown in $\mathrm{t}(4 ; 14) \mathrm{MM}$ cells. Recently a microRNA (miRNA) profiling in $\mathrm{t}(4 ; 14) \mathrm{MM}$ cells identified miR-126* as an MMSET-regulated miRNA, which could specifically target the $3^{\prime}$-untranslated region of c-Myc and inhibit its translation [33]. Moreover, the expression of miR-126* was sufficient to decrease the proliferation rate of $\mathrm{t}(4 ; 14) \mathrm{MM}$ cells. Chromatin immunoprecipitation (ChIP) analysis showed that MMSET binds to the miR-126* promoter along with the KAP1 corepressor and histone deacetylases to repress miR-126* transcription. Through quantitative mass spectrometry analysis we found that overexpression of SLAMF7 (also known as CS1) was associated with MMSET overexpression in $\mathrm{t}(4 ; 14) \mathrm{MM}$ cells [34]. Quantitative RTPCR and ChIP analysis indicated that MMSET might regulate the transcription level of SLAMF7 and be an important functional element for SLAMF7 promoter activity.

Nimura et al. found that MMSET is associated with the cell-type-specific transcription factors Sall1, Sall4, and Nanog in embryonic stem cells (ESCs) and Nkx2-5 in embryonic hearts [29]. These results suggested that MMSET functions together with developmental transcription factors to prevent the inappropriate transcription that can lead to various pathophysiologies. Immunoprecipitation combined with mass spectrometry analysis revealed IQGAP1 and TIAM1 as candidate interacting partners with MMSET, and these interactions were confirmed by coimmunoprecipitation [35].
IQGAP1 and TIAM1 are both involved in the WNT signaling pathway through interaction with $\beta$-catenin protein. Gene expression array and real-time PCR analysis indicated that the expression levels of CCND1, an established downstream target of the $\beta$-catenin/Tcf- 4 complex, were reduced significantly upon MMSET knockdown. ChIP analysis showed that MMSET bounds the promoter region of CCND1 [35]. These results suggested that MMSET may regulate the WNT signaling pathway through interaction with $\beta$-catenin. Yang et al. reported that MMSET is a strong coactivator of $\mathrm{NF} \kappa \mathrm{B}$ by directly interacting with $\mathrm{NF} \kappa \mathrm{B}$ for activation of target genes, including those for interleukin-6 (IL-6), IL-8, vascular endothelial growth factor A (VEGFA), cyclin D, Bcl-2, and survivin, in castration-resistant prostate cancer (CRPC) cells [36]. They also found that MMSET is critical for cytokineinduced recruitment of $\mathrm{NF} \kappa \mathrm{B}$ and acetyltransferase p300 and histone hyperacetylation. Ezponda et al. reported that MMSET could activate TWIST1 to promote an epithelialmesenchymal transition and invasion in prostate cancer [37]. Whether the aforementioned MMSET interacting proteins and downstream targets in ESCs and solid tumors play critical roles in $\mathrm{t}(4 ; 14) \mathrm{MM}$ remains to be determined. The MMSET interacting proteins and downstream targets were summarized in Figure 2.

\section{Potential Therapeutics Targeting MMSET or Its Downstream Signalling}

MMSET overexpression is a universal feature of $t(4 ; 14) \mathrm{MM}$. Furthermore, $t(4 ; 14)$ MM cells rely on MMSET expression for proliferation, survival, and tumorigenicity in vivo. These findings highlight MMSET as an attractive target for the treatment of $t(4 ; 14)$ MM. HMT activity of MMSET is essential for its oncogenic function, so it would seem straightforward to design small molecular inhibitors to target MMSET substrate 
binding pocket. However, very few lead compounds have been published to selectively inhibit MMSET catalytic function. The development of MMSET inhibitors might be hampered by the lack of crystallographic structural information on enzyme-substrate complexes. Recently Cao et al. reported a novel strategy for selective targeting of HMT activity [38]. Instead of directly targeting the catalytic SET domain, they exploit the unique regulation of the MLL1 complex by WDR5 and target MLL1 complex assembly without affecting other MLL family HMTs. As a result, the compound MM-401 shows no inhibition for global H3K4me and little toxicity for normal cells. Transcriptome analyses also confirmed the remarkable selectivity of MM-401, which induces changes in gene expression that are highly correlative with MLL1 gene deletion. MMSET is multidomain protein and several of its partners have been identified, so it is a promising strategy to target MMSET complex assembly for inhibitor development. Numerous HDAC inhibitors are being developed, some of which are at clinical trial stages for MM therapy [39]. These HDAC inhibitors might be promising candidates to inhibit MMSET complex assembly, because MMSET associates with HDACs in a large complex [19,27,31].

Sequence-specific gene silencing with small interfering RNA (siRNA) has transformed basic science research, and the efficacy of siRNA therapeutics toward a variety of diseases is now being evaluated in preclinical and clinical trials [40]. The key therapeutic advantage of using siRNA lies in its ability to specifically and potently knock down the expression of disease-causing genes of known sequence. However, clinical use of siRNAs encounters one of the obstacles: delivery of siRNAs to the appropriate cells. Antibody-mediated delivery is an effective method of targeting siRNA to particular cells [41]. Our study showed that SLAMF7 overexpression in $t(4$; 14) MM was associated with MMSET expression [34]. Thus, it is potential to develop $t(4 ; 14)$ MM targeted therapy by SLAMF7 antibody mediated MMSET siRNA delivery. This therapeutic strategy will achieve two levels of targeting for $\mathrm{t}(4 ; 14)$ MM: tumor cell selective delivery by the SLAMF7 antibody and gene pathway selectivity by the MMSET siRNA. Furthermore, it was found that, similar to the effects of knocking down MMSET, overexpression of miR-126* could inhibit proliferation of $\mathrm{t}(4 ; 14) \mathrm{MM}$ cells in vitro. It will be appealing to test whether expression of miR-126* can cause therapeutic effects in vivo.

\section{Conclusion}

The outcome of MM patients has dramatically improved in recent years and this has been possible essentially due to the introduction of the new active agents thalidomide, bortezomib, and lenalidomide, autologous stem-cell transplantation (ASCT), and improvements in supportive care [42]. The median survival is in excess of 5 years. Nevertheless, $\mathrm{MM}$ is still considered an incurable disease in the vast majority of patients and the classical pattern of evolution of the disease is of subsequent responses/relapses, with each relapse generally being of shorter duration than the previous ones [43]. Therefore, additional potent therapeutic strategies are urgently needed. The translocation $t(4 ; 14)$ is one of the most common translocations in MM and is associated with very poor prognosis. MMSET overexpression characterizes all $t(4 ; 14)$ MM patients, and furthermore MMSET protein is required for $\mathrm{t}(4 ; 14) \mathrm{MM}$ cell survival in vitro and in vivo. The key role of MMSET in $\mathrm{t}(4 ; 14) \mathrm{MM}$ allows speculating that a specific pharmacological therapy, which targets this protein, may constitute a novel approach to the treatment of MM.

\section{Conflict of Interests}

The authors declare that there is no conflict of interests regarding the publication of this paper.

\section{Acknowledgments}

This work was supported by the National Medical Research Council of Singapore (http://www.nmrc.gov.sg/) Grant NMRC/BNIG/2006/2013. Wee Joo Chng is supported by NMRC Clinician Scientist Investigator award. This work was partly supported by the National Research Foundation Singapore and the Singapore Ministry of Education under its Research Centres of Excellence initiative.

\section{References}

[1] M. Rodríguez-Paredes and M. Esteller, "Cancer epigenetics reaches mainstream oncology," Nature Medicine, vol. 17, no. 3, pp. 330-339, 2011.

[2] M. A. Dawson and T. Kouzarides, "Cancer epigenetics: from mechanism to therapy," Cell, vol. 150, no. 1, pp. 12-27, 2012.

[3] P. Angrand, F. Apiou, A. F. Stewart, B. Dutrillaux, R. Losson, and P. Chambon, "NSD3, a new SET domain-containing gene, maps to 8p12 and is amplified in human breast cancer cell lines," Genomics, vol. 74, no. 1, pp. 79-88, 2001.

[4] I. Stec, T. J. Wright, G. J. van Ommen et al., "WHSC1, a 90 kb SET domain-containing gene, expressed in early development and homologous to a Drosophila dysmorphy gene maps in the Wolf-Hirschhorn syndrome critical region and is fused to $\mathrm{IgH}$ in $\mathrm{t}(4 ; 14)$ multiple myeloma," Human Molecular Genetics, vol. 7, pp. 1071-1082, 1998.

[5] N. Huang, E. vom Baur, J. Garnier et al., "Two distinct nuclear receptor interaction domains in NSD1, a novel SET protein that exhibits characteristics of both corepressors and coactivators," The EMBO Journal, vol. 17, no. 12, pp. 3398-3412, 1998.

[6] M. Chesi, E. Nardini, R. S. C. Lim, K. D. Smith, W. Michael Kuehl, and P. L. Bergsagel, "The $t(4 ; 14)$ translocation in myeloma dysregulates both FGFR3 and a novel gene, MMSET, resulting in IgH/MMSET hybrid transcripts," Blood, vol. 92, no. 9, pp. 3025-3034, 1998.

[7] C. G. Garlisi, A. S. Uss, H. Xiao et al., "A unique mRNA initiated within a middle intron of WHSC1/MMSET encodes a DNA binding protein that suppresses human IL-5 transcription," American Journal of Respiratory Cell and Molecular Biology, vol. 24, no. 1, pp. 90-98, 2001.

[8] P. V. Peña, F. Davrazou, X. Shi et al., "Molecular mechanism of histone $\mathrm{H} 3 \mathrm{~K} 4 \mathrm{me} 3$ recognition by plant homeodomain of ING2," Nature, vol. 442, no. 7098, pp. 100-103, 2006.

[9] H. Wu, H. Zeng, R. Lam et al., "Structural and histone binding ability characterizations of human PWWP domains," PLOS ONE, vol. 6, no. 6, Article ID e18919, 2011. 
[10] Z. Huang, H. Wu, S. Chuai et al., "NSD2 is recruited through its PHD domain to oncogenic gene loci to drive multiple myeloma," Cancer Research, vol. 73, pp. 6277-6288, 2013.

[11] M. Štros, D. Launholt, and K. D. Grasser, "The HMG-box: a versatile protein domain occurring in a wide variety of DNAbinding proteins," Cellular and Molecular Life Sciences, vol. 64, no. 19-20, pp. 2590-2606, 2007.

[12] J. J. Keats, C. A. Maxwell, B. J. Taylor et al., "Overexpression of transcripts originating from the MMSET locus characterizes all $\mathrm{t}(4 ; 14)(\mathrm{p} 16 ; \mathrm{q} 32)$-positive multiple myeloma patients," Blood, vol. 105, no. 10, pp. 4060-4069, 2005.

[13] J. Lauring, A. M. Abukhdeir, H. Konishi et al., "The multiple myeloma-associated MMSET gene contributes to cellular adhesion, clonogenic growth, and tumorigenicity," Blood, vol. 111, no. 2, pp. 856-864, 2008.

[14] P. L. Bergsagel and W. M. Kuehl, "Molecular pathogenesis and a consequent classification of multiple myeloma," Journal of Clinical Oncology, vol. 23, no. 26, pp. 6333-6338, 2005.

[15] P. L. Bergsagel and W. M. Kuehl, "Critical roles for immunoglobulin translocations and cyclin D dysregulation in multiple myeloma," Immunological Reviews, vol. 194, pp. 96-104, 2003.

[16] J. J. Keats, T. Reiman, A. R. Belch, and L. M. Pilarski, “Ten years and counting: so what do we know about $\mathrm{t}(4 ; 14)(\mathrm{p} 16 ; \mathrm{q} 32)$ multiple myeloma," Leukemia and Lymphoma, vol. 47, no. 11, pp. 2289-2300, 2006.

[17] J. J. Keats, T. Reiman, C. A. Maxwell et al., "In multiple myeloma, $\mathrm{t}(4 ; 14)(\mathrm{p} 16 ; \mathrm{q} 32)$ is an adverse prognostic factor irrespective of FGFR3 expression," Blood, vol. 101, no. 4, pp. 1520-1529, 2003.

[18] M. Santra, F. Zhan, E. Tian, B. Barlogie, and J. Shaughnessy Jr., "A subset of multiple myeloma harboring the $\mathrm{t}(4 ; 14)(\mathrm{p} 16 ; \mathrm{q} 32)$ translocation lacks FGFR3 expression but maintains an IGH/MMSET fusion transcript," Blood, vol. 101, no. 6, pp. 2374-2376, 2003.

[19] J. Marango, M. Shimoyama, H. Nishio et al., "The MMSET protein is a histone methyltransferase with characteristics of a transcriptional corepressor," Blood, vol. 111, no. 6, pp. 3145-3154, 2008.

[20] J. L. R. Brito, B. Walker, M. Jenner et al., "MMSET deregulation affects cell cycle progression and adhesion regulons in $\mathrm{t}(4 ; 14)$ myeloma plasma cells," Haematologica, vol. 94, no. 1, pp. 78-86, 2009.

[21] E. Martinez-Garcia, R. Popovic, D. Min et al., "The MMSET histone methyl transferase switches global histone methylation and alters gene expression in $\mathrm{t}(4 ; 14)$ multiple myeloma cells," Blood, vol. 117, no. 1, pp. 211-220, 2011.

[22] A. J. Kuo, P. Cheung, K. Chen et al., "NSD2 links dimethylation of histone $\mathrm{H} 3$ at lysine 36 to oncogenic programming," Molecular Cell, vol. 44, no. 4, pp. 609-620, 2011.

[23] A. Kassambara, B. Klein, and J. Moreaux, "MMSET is overexpressed in cancers: link with tumor aggressiveness," Biochemical and Biophysical Research Communications, vol. 379, no. 4, pp. 840-845, 2009.

[24] M. Morishita and E. di Luccio, "Cancers and the NSD family of histone lysine methyltransferases," Biochimica et Biophysica Acta, vol. 1816, no. 2, pp. 158-163, 2011.

[25] X. J. Sun, J. Wei, X. Y. Wu et al., "Identification and characterization of a novel human histone $\mathrm{H} 3$ lysine 36-specific methyltransferase," Journal of Biological Chemistry, vol. 280, no. 42, pp. 35261-35271, 2005.

[26] H. B. Kang, Y. Choi, J. M. Lee et al., "The histone methyltransferase, NSD2, enhances androgen receptor-mediated transcription," FEBS Letters, vol. 583, no. 12, pp. 1880-1886, 2009.
[27] J. Y. Kim, J. K. Hae, N. W. Choe et al., "Multiple myelomarelated WHSC1/MMSET isoform RE-IIBP is a histone methyltransferase with transcriptional repression activity," Molecular and Cellular Biology, vol. 28, no. 6, pp. 2023-2034, 2008.

[28] Y. Li, P. Trojer, C. Xu et al., "The target of the NSD family of histone lysine methyltransferases depends on the nature of the substrate," The Journal of Biological Chemistry, vol. 284, no. 49, pp. 34283-34295, 2009.

[29] K. Nimura, K. Ura, H. Shiratori et al., "A histone H3 lysine 36 trimethyltransferase links Nkx2-5 to Wolf-Hirschhorn syndrome," Nature, vol. 460, no. 7252, pp. 287-291, 2009.

[30] H. Pei, L. Zhang, K. Luo et al., "MMSET regulates histone H4K20 methylation and 53BP1 accumulation at DNA damage sites," Nature, vol. 470, no. 7332, pp. 124-128, 2011.

[31] K. Todoerti, D. Ronchetti, L. Agnelli et al., "Transcription repression activity is associated with the type I isoform of the MMSET gene involved in $\mathrm{t}(4 ; 14)$ in multiple myeloma," British Journal of Haematology, vol. 131, no. 2, pp. 214-218, 2005.

[32] O. Binda, "On your histone mark, SET, methylate!," Epigenetics, vol. 8, no. 5, pp. 457-463, 2013.

[33] D. J. Min, T. Ezponda, M. K. Kim et al., "MMSET stimulates myeloma cell growth through microRNA-mediated modulation of c-MYC," Leukemia, vol. 27, no. 3, pp. 686-694, 2013.

[34] Z. Xie, J. Gunaratne, L. L. Cheong et al., "Plasma membrane proteomics identifies biomarkers associated with MMSET overexpression in T(4;14) multiple myeloma," Oncotarget, vol. 4, no. 7, pp. 1008-1018, 2013.

[35] G. Toyokawa, H. Cho, K. Masuda et al., "Histone lysine methyltransferase Wolf-Hirschhorn syndrome candidate 1 is involved in human carcinogenesis through regulation of the WNT pathway," Neoplasia, vol. 13, no. 10, pp. 887-898, 2011.

[36] P. Yang, L. Guo, Z. J. Duan et al., "Histone methyltransferase NSD2/MMSET mediates constitutive NF-kb signaling for cancer cell proliferation, survival, and tumor growth via a feedforward loop," Molecular and Cellular Biology, vol. 32, no. 15, pp. 3121-3131, 2012.

[37] T. Ezponda, R. Popovic, M. Y. Shah et al., "The histone methyltransferase MMSET/WHSC1 activates TWIST1 to promote an epithelial-mesenchymal transition and invasive properties of prostate cancer," Oncogene, vol. 32, no. 23, pp. 2882-2890, 2013.

[38] F. Cao, E. C. Townsend, H. Karatas et al., "Targeting MLL1 H3K4 methyltransferase activity in mixed-lineage leukemia," Molecular Cell, vol. 53, no. 2, pp. 247-261, 2014.

[39] E. M. Smith, K. Boyd, and F. E. Davies, "The potential role of epigenetic therapy in multiple myeloma," British Journal of Haematology, vol. 148, no. 5, pp. 702-713, 2010.

[40] D. H. Kim and J. J. Rossi, "Strategies for silencing human disease using RNA interference," Nature Reviews Genetics, vol. 8, no. 3, pp. 173-184, 2007.

[41] K. F. Pirollo and E. H. Chang, "Targeted delivery of small interfering RNA: approaching effective cancer therapies," Cancer Research, vol. 68, no. 5, pp. 1247-1250, 2008.

[42] S. V. Rajkumar, "Treatment of multiple myeloma," Nature Reviews Clinical Oncology, vol. 8, no. 8, pp. 479-491, 2011.

[43] M. V. Mateos, E. M. Ocio, and J. F. San Miguel, "Novel generation of agents with proven clinical activity in multiple myeloma," Seminars in Oncology, vol. 40, pp. 618-633, 2013. 


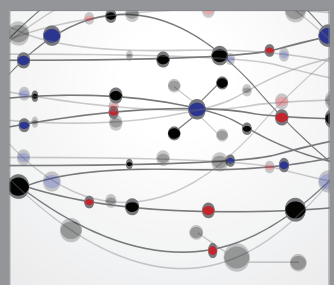

The Scientific World Journal
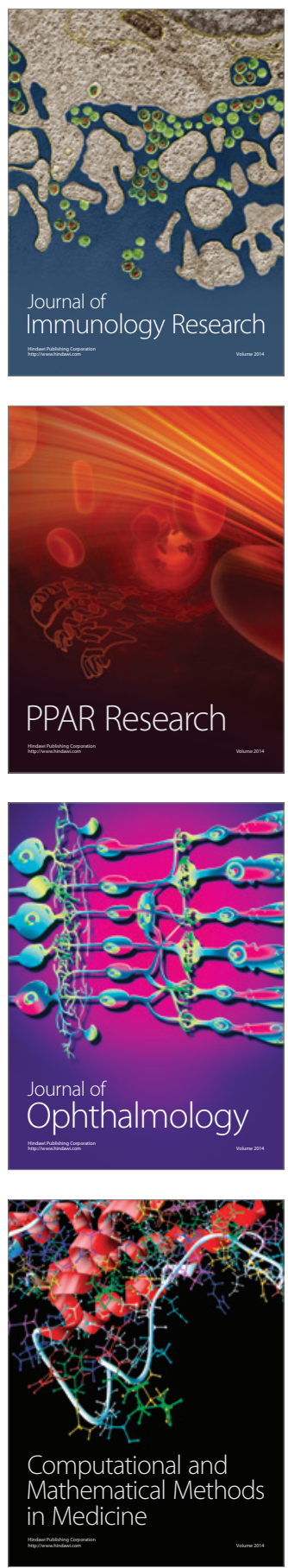

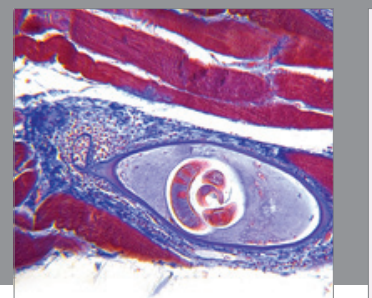

Gastroenterology

Research and Practice
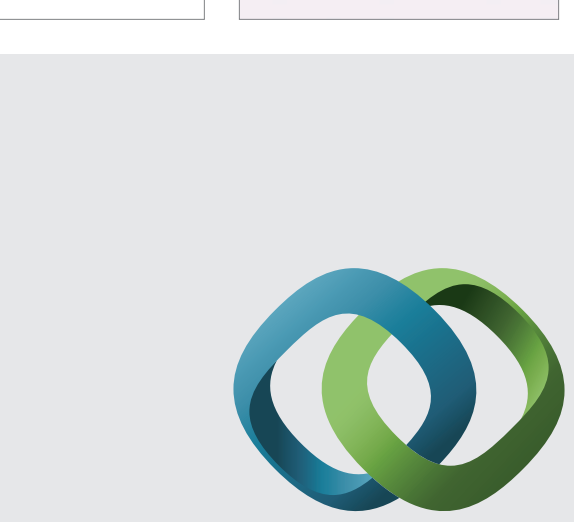

\section{Hindawi}

Submit your manuscripts at

http://www.hindawi.com
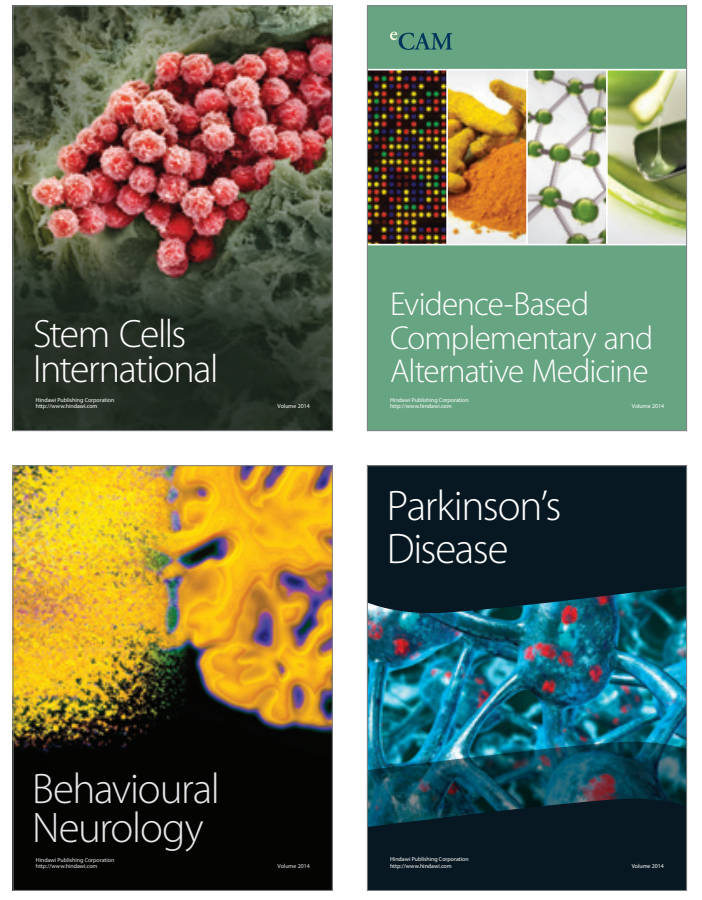
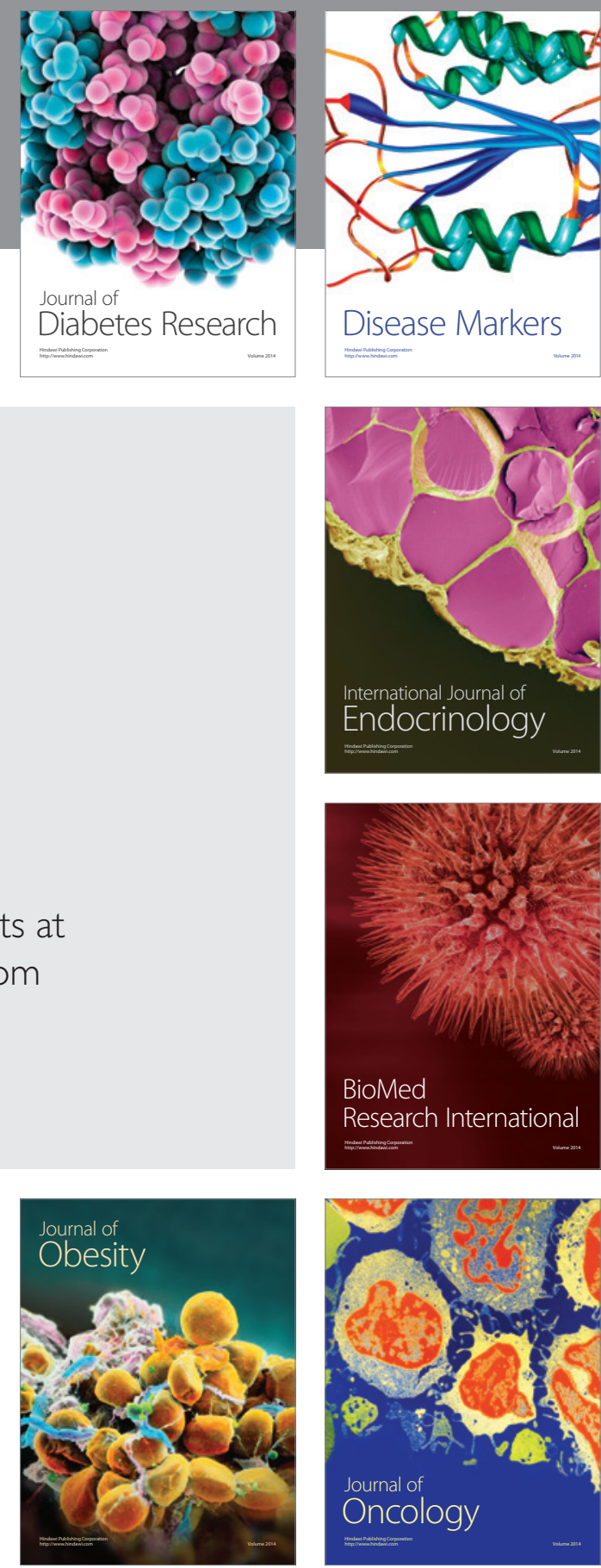

Disease Markers
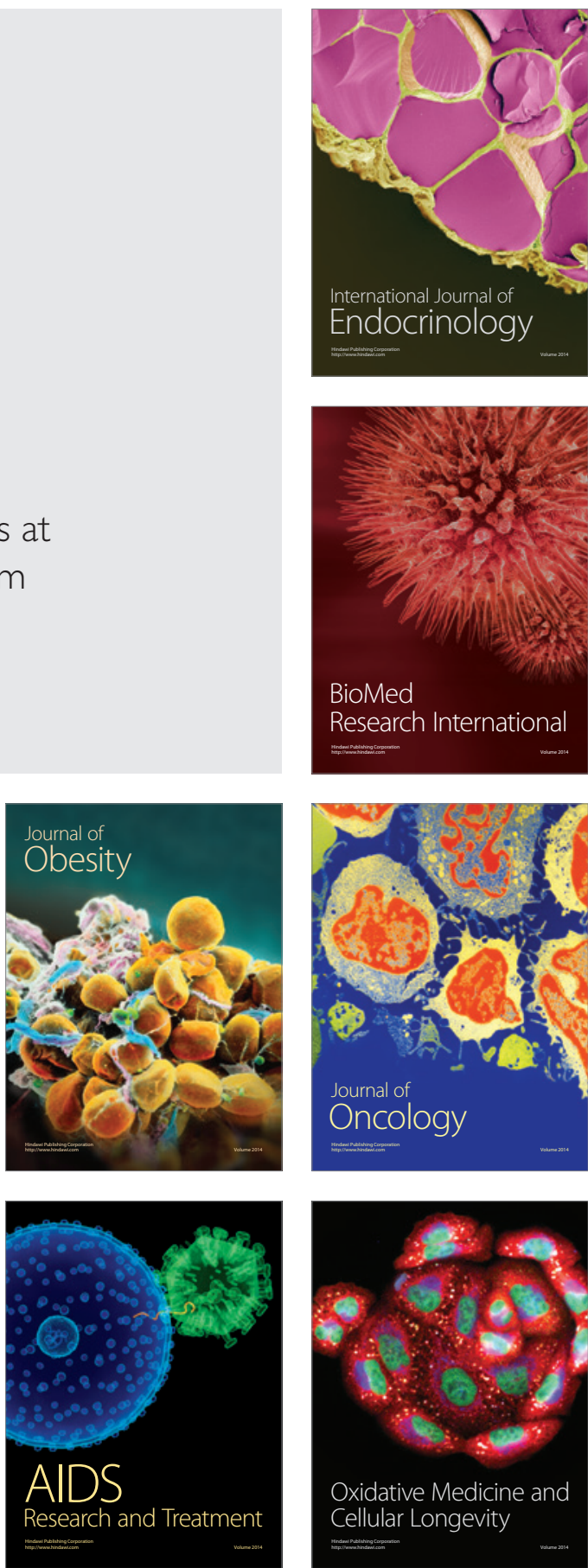